\title{
Prevalence and Antibiogram of Extended Spectrum Beta Lactamases producing Enterobacteriaceae from Urine in Tirunelveli Medical College Hospital
}

\author{
V. P. Sarasu ${ }^{1}$ and K. Girija ${ }^{2 *}$ \\ ${ }^{1}$ Department of Microbiology, Govt. Medical College, Pudukkottai, India \\ ${ }^{2}$ Department of Microbiology, Govt. Thiruvarur Medical College, Thiruvarur, India
}

*Corresponding author

\section{A B S T R A C T}

\section{Keywords}

ESBL, ESBL Hi

Crome agar,

Enterobacteriaceae,

susceptibility

Article Info

Accepted:

20 May 2021

Available Online:

10 June 2021
In the recent years, there has been an increased incidence and prevalence of extended spectrum beta-lactamase (ESBL) producing organisms all over the world and also in various parts of India. ESBL producers pose unique challenges to clinical microbiologist as well as clinicians. As multi drug resistance is rampant, the current study was undertaken to know the prevalence of ESBL producing Enterobacteriaceae in our tertiary health care centre. This study was carried out on 120 urine specimen collected from catheterized patients, aged between 20-70 yrs. The screening for ESBL production was done by the disc diffusion test as recommended by the Clinical and Laboratory Standards Institute(CLSI) and confirmed by the phenotypic disc confirmatory test (PDCT) and ESBL Hi Crome agar(Hi media-Mumbai). 28 specimen were culture positive among 120 samples. Escherichia coli (41.17\%) was the most common isolate, followed by Klebsiella pneumoniae (35.29\%). ESBL production was observed in 15(88.2\%) isolates. The isolates of Escherichia coli (40\%) were the most common ESBL producers, followed by Klebsiella pneumoniae (33.33\%) and others. All isolates were sensitive to imipenem. There is a high prevalence of ESBL production in our hospital. Specific tests to detect ESBL production should be done routinely and an empirical therapy policy should be applied to the high risk units, based on the prevalence of the ESBL producing Enterobacteriaceae.

\section{Introduction}

ESBL producers pose unique challenges to clinical microbiologists as well as clinicians. ESBLs are enzymes capable of hydrolysing penicillins, broad-spectrum cephalosporins and monobactams, and are generally derived from TEM and SHV-type enzymes. ESBLs are often located on plasmids that are transferable from strain to strain and between bacterial species. ${ }^{1}$ ESBLs are more prevalent in Klebsiella pneumonia, Klebsiella oxytoca and Escherichia coli. ${ }^{2}$ Although the prevalence of ESBLs is not known, it is clearly increasing, and in many parts of the world 10-40\% of strains of Escherichia coli and Klebsiella pneumoniae express ESBLs. ${ }^{1}$ Clinical outcomes data indicate that ESBLs 
are clinically significant and, when detected, indicate the need for the use of appropriate antibacterial agents. ${ }^{1}$ Delayed recognition and inappropriate treatment of severe infections caused by ESBL producers with cephalosporin has been associated with increased mortality. ${ }^{3}$ The detection of ESBL expression has proved to be difficult for many laboratories because the resistant ESBL producing organisms appear to be susceptible in the in vitro routine testing and result in treatment failure. ${ }^{4}$ The ready-to-use chromogenic selective medium offers good sensitivity and high specificity, and has the advantages of allowing easy discrimination of different colonies simply according to their colour, which is particularly useful in specimens containing a resident associated flora. ${ }^{5}$ Chromogenic agar for detection of ESBL producers contains several artificial substrates that when hydrolysed by specific bacterial enzymes produce coloured compounds. Koneman et al., states chromogenic agar allowed a $>50 \%$ reduction in inoculation time and a $>20 \%$ reduction in work up time. ${ }^{6}$

In the past few years, the number of catheter associated UTIs caused by resistant Gram negative bacteria has risen. This is mainly due to the spread of ESBL producing bacteria and they are difficult to treat. In the background, this study was done to identify the prevalance and antibiogram pattern of ESBL producing Enterobacteriaceae among urinary isolates and to compare the effectiveness of ESBL HiCrome agar with disc diffusion test (by disc diffusion screening and phenotypic confirmation test as recommended by CLSI).

\section{Materials and Methods}

The present study was conducted at Tirunelveli Medical College, from December 2012 to April 2013.This study was approved by the Ethical committee of Tirunelveli medical college.
Urine samples were collected from 120 catheterised patients in the age group of 20-70 yrs under aseptic precautions. Urine was plated onto Nutrient Agar Plates, MacConkey agar plates and ESBL Hi Crome agar plates. Bacterial isolates were identified by standard procedures. Antibiotic susceptibility testing was done by Kirby Bauer disc diffusion method as per CLSI guidelines. ${ }^{7}$.

ESBL screening test was done using cefotaxime and ceftazidime disc. Isolates were confirmed as ESBL producers by PDCT if there was a $>5 \mathrm{~mm}$ increase in the zone diameter for ceftazidime -clavulanic acid, versus its zone diameter when it was tested by ceftazidime alone. ${ }^{7}$

For ESBL Hi Crome agar, the colour and intensity of the colonies on chrom agar were recorded according to the colour chart provided by the manufacturer.(Escherichia coli -pink to purple; Klebsiella -bluish green). ${ }^{8}$

The quality control strains used when performing the screening and the phenotypic confirmatory tests were a non-ESBLproducing organism (Escherichia coli ATCC 25922) and an ESBL-producing organism (Klebsiella pneumoniae ATCC 700603).

\section{Results and Discussion}

Out of the 120 urine samples, 28 were culture positive. Among the 28 isolates 17 belonged to Enterobacteriaceae family of which 7 were Escherichia coli (57.8\%), followed by 6 Klebsiella pneumoniae (25.6\%) and 4 Klebsiella oxytoca (16.6\%). Of the 17 Enterobacteriaceae isolates, 15 were positive in the ESBL screening test.

The prevalence of ESBL in this study was $53.6 \%$. When these 15 isolates were subjected to the confirmatory test, all $15(100 \%)$ isolates 
were confirmed as ESBL producers by using the PDCT and ESBL Hi Crome agar.

Escherichia coli (6) was the most common ESBL producing Enterobacteriaceae, followed by Klebsiella pneumoniae (5) and Klebsiella oxytoca (4).(Table 1) ESBL was most commonly found in general surgical wards (11 isolates) compared to other wards. Four (4) isolates were from obstetric post-operative wards.(Table 1)

The antibiotic sensitivity pattern of the ESBL isolates revealed that $100 \%$ of the isolates were sensitive to imipenem and cefoperazonesulbactum. $66.66 \%$ were sensitive to amikacin, $66.66 \%$ were sensitive to norfloxacin, $55.55 \%$ were sensitive to nitrofurantoin, $53.33 \%$ were sensitive to gentamicin and $53.33 \%$ were sensitive to cotrimoxazole. (Table 2) In this study the sensitivity, specificity, positive and negative predictive value of ESBLHi Crome agar were $100 \%, 100 \%, 100 \%$ and $100 \%$ respectively, considering PDCT as the gold standard.

Antibiotic resistance has been noted as a serious problem, all over the world. The third generation cephalosporins have been used in a large proportion of patients and resistance even to these antibiotics has been reported. With the spread of ESBL producing strains in hospitals, it is necessary to know the prevalence of ESBL strains.

ESBL was most commonly seen in surgical and obstetric wards. Basavaraj $\mathrm{M}$ et al., reported $73.33 \%$ in surgical ICU and $27.2 \%$ in obstetric wards. ${ }^{9}$ The prevalence of ESBL was high because most of the cases were referred from peripheral centres with severe illness, invasive procedures and profuse use of antibiotics.
Out of the 17 Enterobacteriaceae isolates, majority were Escherichia coli (41.10\%), followed by Klebsiella pneumoniae (35.30\%) and Klebsiella oxytoca $(23.50 \%)$.

This is similar to findings of Krishna S et al., ${ }^{10}$ Of the 17 isolates, 15(53.6\%) were suspected to be ESBL producers based on the screening test. They were confirmed by PDCT. Dalela et $a l$., reported the prevalence of ESBL to be $61.6 \%$. $^{11}$

This study shows that ESBL Hi crome agar agar is a reliable culture medium for screening and presumptive identification of ESBL producing Enterobacteriaceae directly from clinical samples. ${ }^{12}$ Although it is slightly more expensive, it significantly reduces the need for unnecessary confirmation tests and allows significant time reduction.

In this study all (100\%) of isolates were susceptible to carbapenems, also observed by Krishna S et al., in 2012. ${ }^{10}$ However, carbapenems are antimicrobials that are usually kept in reserve in non-life-threatening infections and in non outbreak situations. Because, heavy use of carbapenems may favour the selection of highly resistant Stenotrophomonas maltophilia. 13 So, treatment strategy should be based on the severity and site of the infection and local susceptibility pattern. It is important to note that delay in adequate therapy will lead to adverse outcomes and potentially increased mortality.

Amikacin has been used as an alternative where gentamicin-resistant isolates remain susceptible to it. In our study amikacin showed good activity with $66.66 \%$ isolates being susceptible. $28.5 \%$ were sensitive to gentamicin. 
Table.1 Prevalence of ESBL producing Enterobacteriaceae among urinary isolates

\begin{tabular}{|c|c|c|c|c|c|c|c|c|c|}
\hline \multirow{2}{*}{$\begin{array}{l}\text { Total no } \\
\text { of } \\
\text { specimen }\end{array}$} & \multirow{2}{*}{$\begin{array}{c}\text { Total no. } \\
\text { of } \\
\text { organisms } \\
\text { isolated }\end{array}$} & \multirow{2}{*}{$\begin{array}{c}\% \text { of } \\
\text { positive } \\
\text { isolates }\end{array}$} & \multirow[t]{2}{*}{$\begin{array}{l}\text { Ward wise } \\
\text { distribution }\end{array}$} & \multicolumn{3}{|c|}{$\begin{array}{c}\text { Total no.of } \\
\text { Enterobacteriaceae }\end{array}$} & \multicolumn{3}{|c|}{$\begin{array}{c}\text { Number of ESBL producing } \\
\text { Enterobacteriaceae }\end{array}$} \\
\hline & & & & $\begin{array}{l}\text { Esch. } \\
\text { coli }\end{array}$ & $\begin{array}{l}\text { Kleb. } \\
\text { pneu }\end{array}$ & $\begin{array}{l}\text { Kleb. } \\
\text { oxy }\end{array}$ & $\begin{array}{l}\text { Esch. } \\
\text { coli }\end{array}$ & $\begin{array}{l}\text { Kleb. } \\
\text { pneu }\end{array}$ & $\begin{array}{l}\text { Kleb. } \\
\text { Oxy }\end{array}$ \\
\hline \multirow[t]{5}{*}{120} & \multirow[t]{5}{*}{28} & \multirow[t]{5}{*}{$23.33 \%$} & Medicine & 0 & 1 & 0 & 0 & 0 & 0 \\
\hline & & & Surgery & 2 & 3 & 2 & $2(13.3 \%)$ & $3(20 \%)$ & $2(13.3 \%)$ \\
\hline & & & $\mathrm{OBG}$ & 3 & 1 & 1 & $2(13.3 \%)$ & $1(6.6 \%)$ & $1(6.6 \%)$ \\
\hline & & & $\begin{array}{l}\text { Surgical } \\
\text { ICU }\end{array}$ & 2 & 1 & 1 & $2(13.3 \%)$ & $1(6.6 \%)$ & $1(6.6 \%)$ \\
\hline & & & Total & 7 & 6 & 4 & 6 & 5 & 4 \\
\hline
\end{tabular}

Table.2 Antibiogram of ESBL isolates

\begin{tabular}{|c|c|c|c|}
\hline Drugs & $\begin{array}{c}\text { Escherichia coli (6) } \\
\text { Sensitive \% }\end{array}$ & $\begin{array}{c}\text { Klebsiella pneumoniae(5) } \\
\text { Sensitive \% }\end{array}$ & $\begin{array}{c}\text { Klebsiella oxytoca (4) } \\
\text { Sensitive \% }\end{array}$ \\
\hline Amikacin & 83.33 & 40 & 75 \\
\hline Gentamicin & 66.66 & 20 & 75 \\
\hline Cotrimoxazole & 16.66 & 60 & 75 \\
\hline Nitrofurantoin & 66.66 & 100 & 0 \\
\hline Norfloxacin & 66.66 & 40 & 0 \\
\hline Cefotaxime & 0 & 0 & 0 \\
\hline $\begin{array}{c}\text { Ceftazidime } \\
\text { Ceftriaxone }\end{array}$ & 0 & 0 & 100 \\
\hline $\begin{array}{c}\text { Ceftazime } \\
\text { clavulanate }\end{array}$ & 0 & 100 & 100 \\
\hline Imipenem & 100 & 100 & 100 \\
\hline $\begin{array}{c}\text { Cefoperazone- } \\
\text { sulbactum }\end{array}$ & 100 & 100 & 50 \\
\hline $\begin{array}{c}\text { Piperacillin- } \\
\text { tazobactum }\end{array}$ & 0 & 0 & 0 \\
\hline
\end{tabular}

Aminzadeh, et al., reported $67.6 \%$ sensitivity of amikacin and nine percent sensitivity to gentamicin. $^{14}$ Al-Muharrmi et al., demonstrated that piperacillin/tazobactam or ciprofloxacin in combination with amikacin has almost similar activity against ESBL producing Escherichia coli and Klebsiella pneumoniae, to that of imipenem. The recommendation of combination therapy with amikacin plus either piperacillin/tazobactam or ciprofloxacin in infections caused by
ESBLs is an alternative to carbapenems as empirical therapy when ESBL infection is suspected. ${ }^{15}$

In the present study, around $50 \%$ of the isolates were sensitive to nitrofurantoin and cotrimoxazole. The oral options available for the treatment of catheter associated UTI caused by ESBL producing bacteria are limited, particularly if susceptibility testing indicates concurrent resistance to 
trimethoprim and quinolones. Fosfomycin can be used for treatment of uncomplicated lower UTI as a single-dose therapy and catheter associated UTI for up to 21 days due to limited systemic absorption. Fosfomycin should not be used for pyelonephritis or severe urinary sepsis. A combination of beta lactam and beta-lactamase inhibitors can be used as an alternative. ${ }^{13}$

Routine detection of ESBL will be useful for the clinicians to select the appropriate antibiotics for the treatment of these strains and to take proper precautions to prevent the spread of these resistant organisms.

The management of ESBL requires a multidisciplinary approach. Co-ordinated participation of microbiologists, clinicians, nursing personnel, hospital infection control team is essential. ${ }^{16}$

\section{References}

1. Rupp M E, Fey P D. Extended spectrum beta-lactamase (ESBL)-producing Enterobacteriaceae: considerations for diagnosis, prevention and drug treatment. Drugs 2003;63:353-65.

2. Russo T A, Johnson J R. Diseases caused by Gram negative Enteric Bacilli. In Longo D L, Fauci A S and Kasper D L editors. Harrison's principle of internal medicine, $18^{\text {th }}$ edition. USA:The Mc Graw Hill Companies;2012;1247.

3. Wani. K. A et al., Extended spectrum blactamase mediated resistance in Escherichia coli in a Tertiary Care Hospital. Int J Health Sci (Qassim) 2009; 3: 155-63.

4. Sharma. A et al., ESBL - A continuous diagnostic challenge to clinical microbiology laboratories. Int $j$ applied biol pharmaceutical technol. 2012 ; 3:915.

5. Reglier. $\mathrm{H}$ et al., Performance of chrom id
ESBL a chromogenic medium for detection of enterobacteriaceae producing extended spectrum beta lactamases. $\mathrm{J}$ Med Microbiol 2008;57:310-5.

6. Koneman E W, Allen S D, Janda W M, Schreckenberger P C, Winn W C, editors. The Enterobactericeae. Color atlas and textbook of diagnostic microbiology, 6th edition. Philadelphia: J.B. Lipincott Companies; 2006 ; 283.

7. Clinical and Laboratory Standards Institute. (2007). Performance standards for antimicrobial susceptibility testing; $17^{\text {th }}$ informational supplement. CLSI document M100-S17; 27:37. Wayne, PA, USA.

8. Hi Crome ESBL Agar Base - M 1829. Hi media laboratories. www.hi media.com.

9. Basavaraj. M C, Jyothi. P, Peerapur basavaraj. V. The prevalence of ESBL among Enterobacteriaceae in a Tertiary care hospital of North Karnataka, India. $J$ Clin Diag Res 2011 ; 5:470-5.

10. Krishna. S et al., Emergence of Enterobacteriaceae producing ESBL from urine samples. Discovery life.2012;1:137.

11. Dalela. G. Prevalence of Extended Spectrum Beta Lactamase (ESBL) Producers among Gram Negative Bacilli from Various Clinical Isolates in a Tertiary Care Hospital at Jhalawar, Rajasthan, India. J Clin Diag Res 2012; 6: 182-7.

12. Samra. Z, M. Heifetz, J. Talmor, E. Bain, and J. Bahar. Evaluation of use of a new chromogenic agar in detection of urinary tract pathogens. J Clin Microbiol 1998; 36: $990-4$.

13. Pallett. A, and Kieran hand. Complicated urinary tract infections: practical solutions for the treatment of multiresistant Gramnegative bacteria. Oxford $J$ Med $J$ Antimicrob Chemother 65 :25-33.

14. Aminzadeh. Z, Kashi. M. S, Sha'bani. M. Bacteriuria by extended-spectrum beta- 
lactamase-producing Escherichia Coli and Klebsiella pneumoniae isolates in a governmental hospital in south of Tehran, Iran. Iranian J Kidney Dis 2008; 2:197200.

15. Al-Muharrmi. Z. Antibiotic combination as empirical therapy for extended spectrum beta-lactamase. Oman Med $J$ 2008; 23: 78-81.

16. Bhattacharya. S. ESBL- From Petri Dish to the Patient. Indian J Med Microbiol 2006; 24 :20-4.

\section{How to cite this article:}

Sarasu, V. P. and Girija, K. 2021. Prevalence and Antibiogram of Extended Spectrum Beta Lactamases producing Enterobacteriaceae from Urine in Tirunelveli Medical College Hospital. Int.J.Curr.Microbiol.App.Sci. 10(06): 497-502. doi: https://doi.org/10.20546/ijcmas.2021.1006.053 